Conclusion By building upon previous animal modeling experiences with rabbit, swine, and canine, we are able to develop a specific model for the simulation of endovascular techniques and device deployment in large aneurysm morphologies. This model is currently being used to test the liquid embolic, Neu$\operatorname{roCURE}^{\circledR}$. The model has been approved by the Food and Drug Administration (FDA) for NeuroCURE assessment, with the goal of reducing large aneurysm recurrence rates in patients.

Disclosures T. Becker: 1; C; NIH STTR Phase II (2R42NS097069-02A1). 2; C; Aneuvas Technologies, Inc. 4; C; Aneuvas Technologies, Inc. 6; C; Aneuvas Technologies, Inc. W. Merritt: 2; C; Aneuvas Technologies, Inc. C. Settanni: 1; C; IH STTR Phase II (2R42NS097069-02A1). N. Norris: 1; C; IH STTR Phase II (2R42NS097069-02A1). A. Ducruet: 1; C; IH STTR Phase II (2R42NS097069-02A1). 4; C; Aneuvas Technologies, Inc. M. Preul: 1; C; NIH STTR Phase II (2R42NS097069-02A1). 4; C; Aneuvas Technologies, Inc.

\section{E-203 SHEATHLESS LOW-PROFILE RADIAL ACCESS UTILIZING THE 071 BENCHMARK GUIDE CATHETER FOR COMPLEX ANTERIOR \& POSTERIOR CEREBROVASCULAR INTERVENTIONS: HIGH TECHNICAL SUCCESS/LOW COMPLICATIONS WITH 'RULE OF .8'}

S Satti ${ }^{*}$, T Eden. Neurointerventional Surgery, Christiana Care Health System, Newark, DE

10.1136/neurintsurg-2020-SNIS.234

Introduction/Purpose Two factors limiting first line radial access for complex cerebrovascular interventions include: radial artery diameter and available catheter technology. Our rule for patient selection is to maintain a radial artery/sheath ratio of $\geq .8$, which we used as the primary inclusion criteria for all procedures. We have increasingly attempted sheathless radial access allowing for largest inner and smallest outer diameter. We describe a low cost/simplified approach to direct radial access with a commonly available guide catheter. We then present our experience of transradial cerebrovascular interventions comparing outcomes between procedures performed using a 6F Terumo Slender Sheath/Benchmark guide (OD 2.53 $\mathrm{mm})$ versus direct sheathless radial access with the Benchmark (OD $2.0 \mathrm{~mm}$ ).

Materials and Method Single institution retrospective electronic query of all $6 \mathrm{~F}$ radial access procedures performed between October 2010 and March 2020 access for cerebrovascular intervention using a Penumbra Benchmark 6F guiding catheter. The procedures were divided in Group 1 - 133 pts (sheath) and Group 2- 32 patients (sheathless). Summary Technique for Direct/Sheathless Access

1. Ultrasound guided: Target vessel selection/measurement. "Inclusion: artery diameter/sheath diameter $\geq .8$ )

4F micropuncture/21 g needle: Radial angiogram and Radial cocktail Exchange: Micropuncture sheath/sheathless Benchmark/BER over $.035 \times 180 \mathrm{~cm}$ glidewirePrimary outcomes included: failure of primary access (need to convert to femoral access) and major complications requiring surgery or transfusion. Secondary outcomes included: infection, development of AV fistula, or development of pseudoaneurysm.

Results Procedural success (completion of planned procedure) was achieved in $98.2 \%$ of patients (average of groups 1 and 2), no significant difference. Procedures performed included a wide spectrum of intracranial pathologies, however, aneurysm treatment represented $>60 \%$ of procedures. Success with the primary access site cannulation was $100 \%$ for both Group 1 and Group 2, when using rule of $\geq .8$. No major complications were encountered in either group of patients; no patients required transfusion or emergency vascular surgery for vessel injury/avulsion, digital ischemia, or compartment syndrome. One patient was identified in Group 1 with a symptomatic radial artery-vein fistula underwent successful elective out-patient surgical repair at 12 months.

Conclusion Sheathless radial access using a Penumbra Benchmark 071 guiding catheter can be used for cerebrovascular interventions in both anterior and posterior circulation pathology. High technical success completing index procedure, success with arterial cannulation and low complication rates may be attributed to patient selection/technique (maintaining artery/ sheath ratio of $\geq .8$ ). Direct radial access requires smaller vessel diameter potentially expanding eligible patients with smaller radial artery diameters.

Disclosures S. Satti: None. T. Eden: None.

\section{E-204 NOVEL BLOOD ANALOGUE FOR IN-VITRO NEUROVASCULAR MODELING}

H Sodawalla*, W Merritt, T Becker. Bioengineering Program, Northern Arizona University, FLAGSTAFF, AZ

\subsection{6/neurintsurg-2020-SNIS.235}

Introduction In-vitro models for simulating surgical procedures is a cost-effective way of creating fail-safe surgical protocols. Insights gained from these models can also aid in testing new medical devices and reduce the use of animal models. Traditional glass- and silicone-based in-vitro models use distilled water with surfactant additives for lubricity. However, a new material, carboxymethyl cellulose (CMC) dissolved in distilled water, can mimic the mechanical and rheological properties of blood. We have shown that CMC fluid out-performs distilled water, glycerol mixtures, and bovine blood, in terms of physiological accuracy.

Methods We tested the density and dynamic viscosity of the following blood analogs: CMC, distilled water, glycerol mixture (22 wt \%), bovine blood (Bos taurus). For each material, samples were tested with a $20 \mathrm{~mm}$ cylindrical parallel plate head geometry $(0.25 \mathrm{ml})$ attached to a hybrid rheometer (DHR-2, TA Instruments), set at $37^{\circ} \mathrm{C}$ (human physiological temperature). Three samples of each blood analog were tested and repeated 5 times. A shear rate sweep from 15 to 105 1/s was tested to cover a wide range of physiological blood flow rates. 


\begin{tabular}{|c|c|c|c|c|}
\hline Fluid Type & $\begin{array}{l}\text { Density } \\
\text { (kg/cu.m) }\end{array}$ & $\begin{array}{l}\text { Dynamic } \\
\text { Viscosity } \\
\left(\mathrm{Pa}^{*} \mathrm{~s}\right)\end{array}$ & $\begin{array}{l}\text { Relative Error } \\
\text { of Density against } \\
\text { human blood }\end{array}$ & $\begin{array}{l}\text { Relative Error } \\
\text { of viscosity against } \\
\text { human blood }\end{array}$ \\
\hline Water & 998 & 0.001002 & $5.85 \%$ & $71.67 \%$ \\
\hline $\begin{array}{l}\text { Bovine Blood } \\
\text { (cow) }\end{array}$ & 1045.9 & 0.003 & $1.33 \%$ & $15.19 \%$ \\
\hline $\begin{array}{l}\text { Glycerol mixture } \\
\text { (22\% wt.) }\end{array}$ & 1052.9 & 0.00124 & $0.70 \%$ & $64.95 \%$ \\
\hline 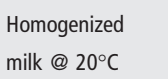 & 1033 & 0.002 & $2.55 \%$ & $43.46 \%$ \\
\hline CMC (1\% wt.) & 1000.4 & 0.003002 & $5.28 \%$ & $15.14 \%$ \\
\hline $\begin{array}{l}\text { Human Blood at } \\
\text { CW }\end{array}$ & 1060 & 0.0035375 & None & None \\
\hline
\end{tabular}

Results We compared the density and dynamic viscosity of different materials in table 1. Different CMC fluid concentrations and the resulting viscosity ranges are compared to blood with increasing shear rate (figure 1). CMC matches the initial viscosity and undergoes shear-thinning like human blood. Distilled water has a lower viscosity than blood that does not change with shear rate. It was found that CMC (1\% wt) is closest to the material properties of human blood flow at the Circle of Willis (CW).

Conclusion CMC fluid is a promising tool in investigating blood flow characteristics in a bench-top model. It can mimic accurate physiologically relevant mechanical properties of blood and has the potential of being used as a go-to tool for medical device testing applications. This fluid will be able to minimize friction between synthetic vessels and endovascular devices and enhance the accuracy of simulated surgery models. Disclosures H. Sodawalla: None. W. Merritt: None. T. Becker: 1; C; NIH STTR Phase I (\#1R41NS097069-01A1).

\section{E-205 CLIP LIGATION OF AN ANTERIOR COMMUNICATING ARTERY ANEURYSM WITH DELAYED RUPTURE AFTER WEB EMBOLIZATION: A CASE REPORT}

N Villelli*, B Bohnstedt. Department of Neurological Surgery, Indiana University, Indianapolis, IN
Introduction The Woven EndoBridge (WEB) aneurysm embolization system has emerged as a new and effective endovascular treatment option for broad necked cerebral aneurysms located at bifurcations. Intra-procedural hemorrhage is a known complication of the device, but we report aneurysmal rupture two days after WEB treatment of an unruptured anterior communicating artery aneurysm. Additionally, instances of surgical clip ligation of aneurysms previously treated with WEB are scarce in the literature. We describe our experience of successful clip ligation of an aneurysm previously treated with WEB.

Methods A 62 year-old female underwent an uneventful WEB embolization of an unruptured anterior communicating artery aneurysm, which measured $3 \mathrm{~mm} \times 4 \mathrm{~mm}$ in maximal diameter. The WEB device used measured $4 \mathrm{~mm} \times 3 \mathrm{~mm}$. Final angiogram demonstrated decreased opacification of the aneurysm dome. The patient was discharged home on post-procedure day 1 on Aspirin $81 \mathrm{mg}$ daily, but returned the following evening with worsening headache. Head CT revealed an acute subarachnoid hemorrhage (SAH) centered on the treated aneurysm. She remained neurologically intact. Formal angiogram after rupture exhibited increased intrasaccular opacification of the aneurysm.

Results The patient was monitored in the ICU and placed on our institution's SAH management protocol including levetiracetam, nimodipine, atorvastatin, intravenous fluids and early DVT prophylaxis. She developed one episode of symptomatic vasospasm requiring intra-arterial milrinone therapy on posthemorrhage day 6. Follow-up angiogram demonstrated continued filling of aneurysm through the WEB device. The device itself appeared to still be under compression, but due to the delayed hemorrhage, further treatment was thought to be appropriate. The patient was subsequently taken to the operating room on post-hemorrhage day 21 for clip ligation of the aneurysm. A standard pterional approach was used. Clip placement was performed without issue and there were no significant challenges faced due to the presence of the WEB device. Post-operative angiogram confirmed aneurysm occlusion. The patient was discharged home on post-operative day 4 , and remained neurologically intact.

Conclusion To our knowledge, this is the first reported case of a delayed aneurysmal subarachnoid hemorrhage after WEB embolization. Additionally, clip ligation of an aneurysm previously treated with WEB can be done safely, and in our patient, did not result in a more challenging operation.

Disclosures N. Villelli: None. B. Bohnstedt: None.

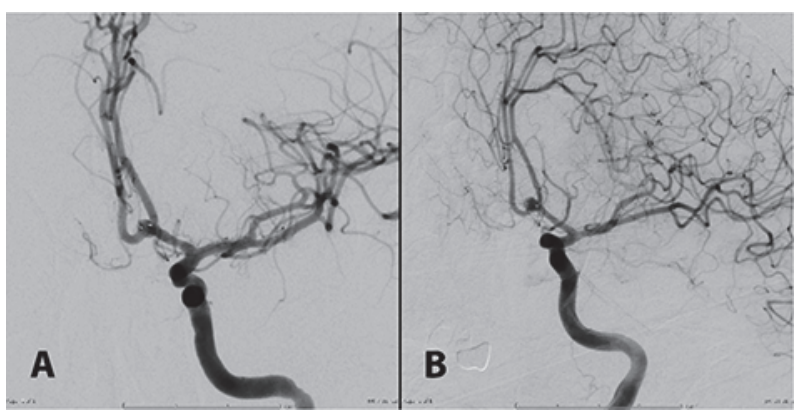

Abstract E-205 Figure 1 A) Initial post-embolization angiogram after WEB deployment demonstrating appropriate placement of the device 